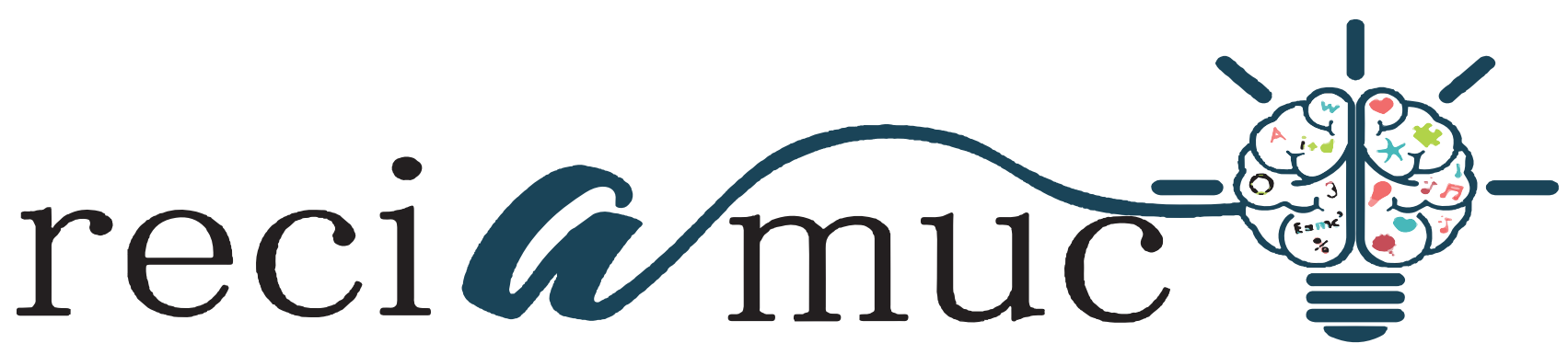

DOI: 10.26820/reciamuc/4.(3).julio.2020.158-166

URL: https://reciamuc.com/index.php/RECIAMUC/article/view/510

EDITORIAL: Saberes del Conocimiento

REVISTA: RECIAMUC

ISSN: 2588-0748

TIPO DE INVESTIGACIÓN: Artículo de Revisión

CÓDIGO UNESCO: 3213 Cirugía; 3213.02 Cirugía Estética

PAGINAS: 158-166

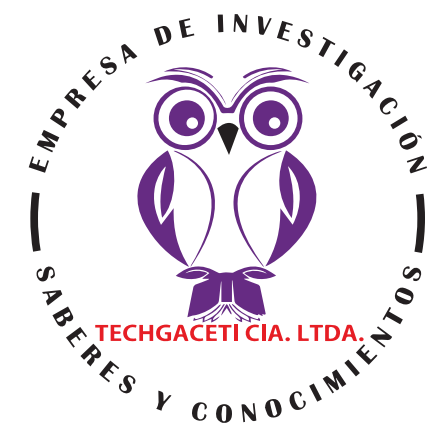

\title{
Nuevas tendencias en la cirugía plástica reconstructiva
}

New trends in reconstructive plastic surgery

Novas tendências em cirurgia plástica reconstrutiva

\section{Fausto Jacinto Loor Valenzuela'; Silvia Esther Rivas Cáceres; Paula Cristina Chang Alvarado; Alfredo Enrique Galindo Veliz ${ }^{4}$}

RECIBIDO: 20/05/2020 ACEPTADO: 19/07/2020 PUBLICADO: 30/07/2020

1. Médico; Investigador Independiente; Guayaquil, Ecuador; loor.faust@gmail.com; (D) https://orcid.org/0000-0002-93061771

2. Médico; Investigador Independiente; Guayaquil, Ecuador; silvitasther9@gmail.com; (iD) https://orcid.org/0000-0003-47240602

3. Médico; Investigador Independiente; Guayaquil, Ecuador; paulachangmd@gmail.com; (iD https://orcid.org/0000-00033215-3856

4. Médico; Investigador Independiente; Guayaquil, Ecuador; dr.galindovelizalfredo@gmail.com; (iD https://orcid.org/00000002-5602-7689

CORRESPONDENCIA

Fausto Jacinto Loor Valenzuel

loor.faust@gmail.com

Guayaquil, Ecuador

(C) RECIAMUC; Editorial Saberes del Conocimiento, 2020 


\section{RESUMEN}

La cirugía plástica ha sido valorada desde el ámbito más banal de la disciplina médica, pareciera existir la necesidad de defenderla puesto que se toma, en algunos casos, como innecesaria e incluso descartable para valorar el estado de salud de los pacientes. Hoy en día un "retoque" facial, lipoescultura, prótesis mamarias, definición abdominal no se limita siquiera al presupuesto o al sexo, resulta común y hasta un logro mostrar. La discusión sobre de la necesidad de la cirugía reconstructiva continúa, implica una percepción de la estética y las expectativas del paciente. Se pretende en esta revisión encontrar las publicaciones científicas que lleven al resumen o caracterización del entorno actual de la disciplina. Pretende responder interrogantes como ¿qué, por qué, para qué y cómo se está haciendo en el mundo en cirugía reconstructiva? Resultan más de 7600 trabajos de los cuales se realiza un primer filtro por tiempo y tipo de publicación. Se escogieron las publicaciones de revistas científicas desde 2015 a la actualidad sin que el tiempo de la publicación sea limitante. La tendencia en las publicaciones sobre cirugía plástica reconstructiva, más allá de direccionarse a procedimientos, destaca la discusión moral y ética de las intervenciones. El medico de esta rama debe enfrentarse a los señalamiento antes que a la técnica. Independientemente de la técnica, podemos concluir que, como hemos ido aprendiendo, estamos frente una nueva realidad que afecta un espectro amplio de la vida que conocíamos, entonces la tendencia en la disciplina medica por ahora y en lo que resta del año será ética, moral, psicológica más que científica.

Palabras clave: Cirugía plástica reconstructiva, tendencia, bioética, tecnología.

\section{ABSTRACT}

Plastic surgery has been valued from the most banal field of medical discipline, there seems to be a need to defend it since it is taken, in some cases, as unnecessary and even disposable to assess the health status of patients. Today a facial "retouch", liposculpture, breast prostheses, abdominal definition is not even limited to budget or sex, it is common and even an achievement to show. The discussion about the need for reconstructive surgery continues, it implies a perception of the aesthetics and expectations of the patient. It is intended in this review to find the scientific publications that lead to the summary or characterization of the current environment of the discipline. It aims to answer questions such as what, why, for what and how is reconstructive surgery being done in the world? There are more than 7600 jobs of which a first filter is made by time and type of publication. The publications of scientific journals from 2015 to the present were chosen without limiting the time of publication. The trend in publications on reconstructive plastic surgery, beyond addressing procedures, highlights the moral and ethical discussion of the interventions. The doctor of this branch must face the signals before the technique. Regardless of the technique, we can conclude that, as we have been learning, we are facing a new reality that affects a wide spectrum of life that we knew, so the trend in medical discipline for now and for the rest of the year will be ethical, moral, psychological rather than scientific.

Keywords: Reconstructive plastic surgery, trend, bioethics, technology.

\section{RESUMO}

A cirurgia plástica tem sido valorizada desde o campo mais banal da disciplina médica, parece haver necessidade de defendê-la, uma vez que é tida, em alguns casos, como desnecessária e até descartável para avaliar o estado de saúde dos pacientes. Hoje um "retoque" facial, lipoescultura, prótese mamária, definição abdominal não se limita nem ao orçamento ou ao sexo, é comum e até uma conquista de se mostrar. A discussão sobre a necessidade da cirurgia reconstrutiva continua, isso implica uma percepção da estética e expectativas do paciente. Pretende-se nesta revisão encontrar as publicações científicas que conduzam à síntese ou caracterização do ambiente atual da disciplina. Tem como objetivo responder a questões como o que, por que, para quê e como a cirurgia reconstrutiva está sendo realizada no mundo? São mais de 7600 trabalhos dos quais é feito um primeiro filtro por tempo e tipo de publicação. As publicações em revistas científicas de 2015 até o presente foram escolhidas sem limitação do tempo de publicação. A tendência de publicações em cirurgia plástica reconstrutiva, além de abordar procedimentos, evidencia a discussão moral e ética das intervenções. O médico deste ramo deve enfrentar os sinais antes da técnica. Independentemente da técnica, podemos concluir que, à medida que vamos aprendendo, estamos diante de uma nova realidade que afeta um amplo espectro de vida que conhecíamos, então a tendência da disciplina médica para agora e para o resto do ano será éticos, morais, psicológicos em vez de científicos.

Palavras-chave: Cirurgia plástica reconstrutiva, tendência, bioética, tecnologia. 


\section{Introducción}

La cirugía plástica ha sido valorada desde el ámbito más banal de la disciplina médica, pareciera existir la necesidad de defenderla puesto que se toma, en algunos casos, como innecesaria e incluso descartable para valorar el estado de salud de los pacientes.

- - En medio de la evolución de la sociedad cada vez es más aceptada la intervención médica por estética. El culto al cuerpo y a la imagen es notorio con la crecida exposición del ser humano entre redes sociales y el impulso a la aceptación de las diferencias. Hoy en día un "retoque" facial, lipoescultura, prótesis mamarias, definición abdominal no se limita siquiera al presupuesto o al sexo, resulta común y hasta un logro mostrar.

Sin embargo, la discusión ética continúa, pues la cirugía plástica no se limita a ello, sino que transforma la calidad de vida de aquel que padece secuelas de alguna enfermedad o muestran defectos congénitos.

En términos generales, se define a la Cirugía Plástica, Estética, Reparadora o Reconstructiva, como la especialidad quirúrgica encargada de restablecer la integridad anatómica o funcional del cuerpo humano, alterado por defectos físicos, congénitos o adquiridos. (Acerbi Cremades, 2009)

Arraiga (2016) expone que "se puede moldear o transformar un defecto, actuando por lo tanto en un sujeto enfermo, que necesita ese acto quirúrgico, similar a otras cirugías necesarias (cáncer de tiroides p ej.) y esta vertiente tiene sus propias implicancias éticas, ya que se pretende el mejor bien para el paciente (beneficencia), se respeta su autonomía, y porque se brinda en el contexto que el paciente solicita o está de acuerdo con esta corrección. Es una cirugía necesaria para la salud del paciente". (Arriagada S., 2016)

La discusión sobre de la necesidad de la cirugía reconstructiva continúa, implica una percepción de la estética y las expectativas del paciente. Se pretende en esta revisión encontrar las publicaciones científicas que lleven al resumen o caracterización del entorno actual de la disciplina. Pretende responder interrogantes como ¿qué, por qué, para qué y cómo se está haciendo en el mundo en cirugía reconstructiva?

\section{Metodología}

A través de las herramientas web se ha realizado una búsqueda de las últimas publicaciones sobre cirugía plástica reconstructiva utilizando la frase como palabras claves. Resultan más de 7600 trabajos de los cuales se realiza un primer filtro por tiempo y tipo de publicación. Se escogieron las publicaciones de revistas científicas desde 2015 a la actualidad sin que el tiempo de la publicación sea limitante pues se consideran publicaciones de data larga siempre que su valor científico no tenga sustituto más actualizado. Los aportes más resaltantes encontrados se plasman como resultados.

\section{Resultados}

En el Siglo XXI, la especialidad quirúrgica ha tomado un verdadero auge. Se han alejado todos los prejuicios pasados y si antes los pacientes que habían recibido sus beneficios, se callaban o lo ocultaban, en la actualidad, pregonan, vanaglorian y enfatizan los logros alcanzados.

Ya no es una cirugía para las elites, ni tampoco solo para mujeres. Por el contrario, se practica en los hospitales públicos; es para ambos sexos; sin límite de edad y variados recursos económicos.

Por estas razones, nadie carga con el peso de los defectos que pueden ser causa del aislamiento del individuo ante su familia, sociedad o ámbito laboral en el que se desempeña. (Acerbi Cremades, 2009)

Cirugía de la imagen. Cosmética, se dice, la única que opera "pacientes sanos" y a la que por tanto no le resulta perdonable po- 
ner a prueba la salud y menos la vida. ¿Hay razón total en esto? ¿Volvemos a la vieja discusión? ¿Son "sanos" los pacientes de cirugía estética? No, a la luz del concepto generalizado por la Organización mundial de la Salud (OMS) desde 1948: "Salud es un estado de completo bienestar, físico, mental y social, no solamente la ausencia de enfermedad". (Díaz R., 2017)

Los procedimientos más comunes en materia de cirugía plástica reconstructiva según Patiño y otros (2018) son la reconstrucción del seno, la reducción de pecho, reconstrucción de la areola y del pezón, cirugías para pies y manos afectadas por cualquier enfermedad, incluyendo tumores (cancerígenos y no cancerígenos), dedos palmeados (cuando los dedos están unidos por una membrana), cirugías faciales como labio leporino y microcirugías reconstructivas como trasplante de piel, grasa, músculo o hueso a la cara con el objetivo de reconstruir cualquier estructura facial o cervical lesionada, incluyendo la mandíbula, lengua, nariz, mejilla, cuero cabelludo. (Patiño Z., Cedeño G., Sánchez V., \& Berruz A., 2018)

Los pacientes con padecimientos relacionados con la Cirugía Plástica serían los que muestran malformaciones congénitas en cara o extremidades, secuelas de quemaduras, secuelas de cáncer de mama o secuelas de alguna lesión traumática. (VaIlarta, Morales, \& A, 2015)

Contrario a los que hoy se percibe los prejuicios y tabús moralistas no han sido enterrados. Las complicaciones, malos resultados y fatalidades aún son interpretados como "castigos" resultantes de la vanidad del paciente o la codicia del cirujano. Los riesgos inherentes a toda práctica médico-quirúrgica, en particular las muertes, provocan un temor y rechazo desmedido tan solo por referirse a intervenciones en la rama reconstructiva al punto de resultar en leyes que prohíben de la cirugía plástica para menores. Igual que todo acto médico, cada caso plástico pone a paciente y ciru- jano, frente a sus deberes morales. Hacer lo correcto. Campo de la ética, materia fundamental que no parece haber acompañado el viaje de la técnica, ni recibido igual atención general. (Díaz R., 2017)

La discusión moralista, ética que plante Díaz (2017) abarca entonces las respuestas que el cirujano también debe resolver como parte de su tarea de brindar salud al paciente "¿Es operar la mejor opción? ¿Hasta dónde invadir química e instrumentalmente? ¿Cuánto trauma causar? ¿Cuánto riesgo asumir? Primum non nocere. La vida es el valor y su valor subsidiario la salud... Pero en las condiciones ambientales (culturales) presentes, el no hacer nada, el rechazar, no "sana" los pacientes, no garantiza su seguridad, ni exime la conciencia de los cirujanos... Ahí está el problema..." (Díaz R., 2017)

Para Bozola (2008) se tiene que intentar siempre la menor y la mejor cicatriz posible. Esta aseveración implica parte importante de las técnicas y materiales utilizados en la cirugía plástica, pues el resultado será medido también por las marcas que deja, en este caso la cicatriz. El cirujano debe orientar sus esfuerzos en minimizar el impacto que dejará la cirugía por ende debe promover los cuidados necesarios para que la apariencia sea lo más imperceptible. (Dieppa Ramírez \& Alba Matos, 2013)

Los estudios y publicaciones recientes más populares en cuanto a la cirugía plástica reconstructiva abordan principalmente cirugías en cirugía mamaria, los injertos, el uso del plasma rico en plaquetas y biotecnología aplicada a la cirugía plástica.

De estas publicaciones se puede resaltar las discusiones y conclusiones principales

Publicación: Cierre de lesiones en pacientes con pie diabético por injerto de Davis. Año 2017

El auto-injerto de piel es una de las alternativas disponibles en cirugía reconstructiva 
para restaurar de forma definitiva la barrera cutánea frente a heridas de diversas etiologías donde es imposible realizar un cierre primario. Ellos deben realizarse en las siguientes situaciones para optimizar los resultados: en áreas expuestas a una infección con gran cantidad de pérdida de piel, en quemaduras extensas, por razones estéticas, en cirugías reconstructivas por daño de la piel o pérdida de la misma (como en los tumores de piel), en cirugías donde las heridas no pueden suturarse directamente y en heridas muy extensas o úlceras de diferente etiología.

Es posible clasificarlos de acuerdo al donante, al espesor, y a su origen en:

Según donante en:

- Autólogo: cuando es del propio paciente.

- Homólogo: cuando es de un donante vivo.

- Aloinjerto: cuando provienen de un donante cadáver.

- Heterólogo o xenoinjerto: cuando proviene de un animal (cerdo y otros).

Según espesor:

- Piel total (Wolfe-Krause): incluyen dermis y epidermis completas. Las zonas de elección son el hueco supraclavicular, el pliegue inguinal, codo, y muñeca. El resultado estético de este tipo de injerto es mejor que el de piel parcial, pero los injertos deben ser pequeños para poder realizar un cierre primario del sitio donante.

- Piel parcial (Ollier-Tersch): incluyen a la epidermis y grosores variables de dermis, y pueden ser sub-clasificados en: delgados, intermedios y gruesos $(0,30$ a $0,45 \mathrm{~mm}$ ). Las zonas de obtención frecuentes son los muslos, los glúteos, y el cuero cabelludo, y pueden tomarse de otras zonas en caso de necesidad.
Según el origen :

- Dermo-epidérmicos, nervio, tendón, cartílago, hueso y compuestos (toman varios tipos de tejidos).

Cabe señalar que los injertos de Davis son un tipo especial de autoinjerto cutáneo, que se realiza con la piel en forma de pequeños discos de alrededor de tres miímetros de diámetro. En su centro estos discos son más gruesos y adelgazan hacia la periferia, de forma tal que comparten algunas características de los injertos de piel de espesor parcial y total.1 (Bravo Y., Fillor V., \& Valdés P., 2017)

El injerto de Davis puede fracasar por un lecho receptor mal irrigado, la infección y la movilización de los injertos por una curación inadecuada. Hay que agregar la desventaja que posee de un pobre resultado estético tanto a nivel del área dadora de los injertos como a nivel del lecho injertado. (Bravo Y., Fillor V., \& Valdés P., 2017). El pobre resultado estético es una consideración secundaria al tratarse de pacientes con una elevada probabilidad de perder la extremidad sin que ello represente una desestimación de la estética, en esos casos priva la expectativa del paciente y la capacidad humana del médico tratante para enfocarse en los beneficios que proporciona la intervención.

La mayor resistencia a traumas, prendimiento fácil, escaza retracción secundaria, buen resultado funcional, se puede realizar con anestesia local y de forma ambulatoria, lo que se traduce en una reducción de los gastos hospitalarios, además de constituir una buena opción para aquellos pacientes que necesiten de cobertura cutánea por distintas etiologías con baja morbilidad y excelentes resultados son las ventajas que el autor recoge con este tipo de injertos.

Por tanto concluye que el injerto de piel por el método de Davis constituye una buena opción para aquellos pacientes portadores de pie diabético que necesiten de cobertura cutánea con baja morbilidad y excelen- 
tes resultados en aquellos pacientes en los que no se pueda realizar un colgajo en primera instancia. (Bravo Y., Fillor V., \& Valdés P., 2017)

Publicación: Utilización de colgajo radial para cobertura de exposición protésica en craneoplastias. Año 2020

La craneoplastia es un procedimiento necesario para cubrir defectos craneales luego de resección ósea por distintas etiologías, como hemorragia intracraneal, traumatismos craneoencefálicos, tumores o infecciones. Una de las complicaciones frecuentes es la exposición de placas de craneoplastia por dehiscencia de herida cutánea. Estas son complicaciones frecuentes y frustrantes para el paciente y el cirujano plástico. La transferencia de tejidos a distancia brinda una solución para estos pacientes. El colgajo radial antebraquial reúne las condiciones necesarias para la cobertura frente a los diversos materiales son los que se utilizan en las craneoplastias incluidos hueso autólogo, mallas de titanio y polimetilmetacrilato (PMMA). En este trabajo los autores se proponen un abordaje del paciente conservador, asi lo expresan debido a que la extracción de la placa presenta implicancias psicológicas, económicas y estéticas de interés en el paciente. Pretenden demostrar que, mediante la transferencia libre de tejido, se pueden tratar este tipo de pacientes. (Rocca, Mackfarlane, Artero, Martínez, \& Pefaure, 2020)

En casos de defectos de cobertura cutáneos secundarios a exposición de material protésico por cranioplastia, es necesaria la cobertura con un tejido bien vascularizado, que generalmente corresponde a la transferencia libre. Es necesario identificar el pedículo vascular receptor previo a la toma de la decisión de la transferencia.

Concluyen que "Es necesario identificar el pedículo vascular receptor previo a la toma de la decisión de la transferencia. Los vasos faciales homolaterales son la prioridad para dicha transferencia. El colgajo antebraquial a flujo directo o reverso cumple las necesidades para dicha cobertura. Es prioritaria la cobertura de toda la unidad estética para obtener mejores resultados estéticos. No está claro si debe resecarse o no el material protésico en el primer tiempo ya que es posible que la transferencia de tejido libre vascularizado actúe sellando el espacio entre el material protésico y tejidos blandos". (Rocca, Mackfarlane, Artero, Martínez, \& Pefaure, 2020)

Publicación: Aplicaciones del plasma rico en plaquetas en cirugía estética: revisión de la literatura. Año 2020

El plasma rico en plaquetas es un derivado sanguíneo concentrado, utilizado como terapia regenerativa, lo cual esta mediado por factores de crecimiento que favorecen el recambio y regeneración celular Luego de una revisión bibliográfica concluye que el plasma rico en plaquetas ha atraído la atención en varios campos quirúrgicos, en especial el de la cirugía estética, debido a la amplia variedad de beneficios clínicos potenciales que se observan tras su aplicación. Se informan resultados positivos en el rejuvenecimiento de la piel, en la alopecia, en daños traumáticos y resección tumoral, pues mejora la supervivencia de los injertos, en las cicatrices del acné, así como en la cirugía estética de las mamas. El tratamiento con plasma rico en plaquetas con fines estéticos se ha mostrado con un futuro brillante para una intervención cosmética segura y eficiente. Sin embargo, se necesitan más estudios para mejorar la comprensión de las limitaciones y los beneficios en las fases clínicas (Escobar vega, Zaldain Ramos, Novo, Tamayo Carbon, \& Exposito Jalturin, 2020)

Publicación: Aplicaciones de la impresión 3D en cirugía plástica reconstructiva Año 2020

La impresión en tres dimensiones, también conocida como "manufactura aditiva", es una tecnología que permite la construcción de modelos físicos diseñados por compu- 
tadora, un concepto que hasta hace unos años parecía inalcanzable. La manufactura aditiva permite fabricar modelos físicos basados en diseños creados en una computadora (4), la cual transmite información a una impresora especializada en la cual se agregarán capas sucesivas de material hasta obtener el objeto deseado. (Telich-Tarriba, Ramírez-Sosa, Palafox, Ortega-Hernández, \& Rendón-Medina, 2020)

En el campo de la cirugía plástica la aplicación de esta herramienta es amplia, abarca desde la oportunidad de generar nuevas experiencias educativas para los especialistas en entrenamiento, hasta reproducir en modo fidedigno la anatomía de los pacientes, y permitir a los cirujanos planificar y practicar procedimientos. La manufactura aditiva permite exportar estos estudios a una computadora para generar una representación tangible de las estructuras anatómicas del paciente.(Telich-Tarriba, Ramírez-Sosa, Palafox, Ortega-Hernández, \& Rendón-Medina, 2020)

Una de las primeras áreas en adoptar estas tecnologías tridimensionales fue la cirugía craneofacial, ya que se logra determinar las dimensiones, posiciones y angulaciones de distintas estructuras de una forma precisa, permitiendo planificar procedimientos complejos o reconstrucciones en pacientes con padecimientos poco comunes como la presencia de fisuras faciales, craneosinostosis sindrómicas y otras malformaciones congénitas. También tiene la capacidad de simular procedimientos sobre estos modelos lo que potencialmente reduce la incidencia de complicaciones, mejora los desenlaces y permite reducir los tiempos quirúrgicos. (Telich-Tarriba, Ramírez-Sosa, Palafox, Ortega-Hernández, \& Rendón-Medina, 2020)

La utilidad de la impresión 3D va más allá de la planificación preoperatoria, ya que se trata de una herramienta que nos brinda la posibilidad de crear objetos que guíen la realización de un acto quirúrgico, permitiendo nos acelerar y facilitar la realización de diversos procedimientos. Mediante la creación de implantes 3D de titanio que ajustan fielmente a la morfología del defecto, creados en base a los estudios de imagen específicos de cada paciente, en ello radica importancia de la utilización de implantes personalizados porque se logra optimizar los resultados durante el transoperatorio. (Telich-Tarriba, Ramírez-Sosa, Palafox, Ortega-Hernández, \& Rendón-Medina, 2020)

Publicación: Desarrollo y validación de modelos de simulación en cirugía plástica. Año 2020

En el "presente" como fracción de tiempo desconocido, el ejercicio de la medicina enfrenta la diferenciación entre arte, o ciencia y biotecnología. La sociedad moderna cree que la medicina es una ciencia. Los medios de comunicación, los pacientes, los jueces y hasta los médicos mismos apelan al científico y a la ciencia perdiendo por completo la dimensión del médico como ser humano facilitador o promotor de la salud y la vida. (Mejía, 2020)

La tecnología y la biotecnología pertenecen al orden del instrumento, la herramienta, para el ejercicio de un arte cuya incerteza se mide con probabilidades, definidas matemáticamente sobre datos empíricos que la ciencia busca validar. El acto médico -no biotecnológico-, es interpretación casi mágica de un mundo ajeno, igualmente complejo e incierto. El acto médico biotecnológico es corte, sección, simplificación, de una fracción infinitesimal del individuo, sin embargo, es lo que todos creen que la medicina y sus especialidades son: biotecnología, determinismo y acierto o error. La biotecnología es, un apoyo fundamental del ejercicio médico moderno, sin embargo, involucra en sí misma el poder para reducir la atención médica al dato fragmentado, aislado, frío y sin valor. El ejercicio de la medicina se hace por el hombre y para el hombre con la intención objetiva de apoyar su bienestar físico, psíquico afectivo y ambiental. (Mejía, 2020) 


\section{Conclusiones}

La tendencia en las publicaciones sobre cirugía plástica reconstructiva, más allá de direccionarse a procedimientos, destaca la discusión moral y ética de las intervenciones. El medico de esta rama debe enfrentarse a los señalamiento antes que a la técnica.

Contrario a la premisa inicial, la expectativa era conseguir nuevas prácticas, nuevos ensayos, quizá los que apuntan a la cirugía reconstructiva luego de alguna complicación por procedimientos estéticos mal aplicados. Gratamente hemos encontrado trabajos en donde se trata de simplificar los procedimientos en pro del bienestar del paciente.

Lo novedoso de ésta publicaciones o el interés de la investigación apunta a la biotecnología, a reducir el riesgo y los impactos negativos sobre el paciente, quizá a disminuir el margen de error propio del ser humano que realiza el procedimiento por más que lo haga de forma pulcra.

La ciencia médica va de la mano con el desarrollo científico y tecnológico, a medida que van surgiendo avances es posible sumergirse a desarrollar técnicas que mejoren la forma en que se practica la medicina, ya sea con la creación métodos diagnósticos, enfocados cada vez más a niveles moleculares, o el surgimiento de materiales y dispositivos que nos permitan trabajar a un nivel macroscópico y tangible.

Dice Teich y otros (2020):

La impresión 3D pertenece a este nuevo capítulo de la era industrial y tecnológica con un elevado potencial de ser utilizada en una amplia gama de áreas en la medicina... la aplicación de la impresión 3D en cirugía reconstructiva va mucho más allá de la creación de estructuras aplicables al paciente en las distintas etapas de su valoración y tratamiento, ... también se ha convertido en una verdadera fuente de posibilidades para la enseñanza, es una tecnología revo- lucionaria que si bien no está regulada al cien por ciento, resulta ser accesible y económicamente sustentable. (Telich-Tarriba, Ramírez-Sosa, Palafox, Ortega-Hernández, \& Rendón-Medina, 2020)

Sin embargo, los avances en el área no apuntan solo a tecnología vista desde aparatos, sino también a la utilización de opciones biológicas cuya técnica proporciona comprobados resultados menos invasivos e igual de exitosos, referimos el caso del uso del plasma rico en plaquetas.

Finalmente, el proyecto es el mismo, el menor riesgo para conseguir el resultado más discreto, devolviendo una funcionalidad deseable para el afectado visualizando las menores cicatrices posibles.

Para este año, existe un acercamiento a cómo solucionar las intervenciones reconstructivas programadas en tiempos pandémicos como los que enfrentamos a la fecha. Y no solo eso, sino también de que manera continua la preparación de los aspirantes en cirugía plástica reconstructiva en momentos donde el distanciamiento social demuestra ser una práctica contundentemente efectiva para controlar los contagios del complejo virus COVID-19.

Independientemente de la técnica, podemos concluir que, como hemos ido aprendiendo, estamos frente una nueva realidad que afecta un espectro amplio de la vida que conocíamos, entonces la tendencia en la disciplina medica por ahora y en lo que resta del año será ética, moral, psicológica más que científica.

\section{Bibliografía}

Acerbi Cremades, N. (2009). Orígenes de la cirugía plástica. Padres, pioneros y otros más. Revista de Salud Pública, XIII(2), 47-52.

Arriagada S., J. (2016). Buenas prácticas en cirugía estética: Algunas consideraciones desde la bioética. Revista médica Clínica Los Condes, 27(1), 113-121.

Bravo Y., C., Fillor V., F., \& Valdés P., C. (2017). Cie- 
rre de lesiones en pacientes con pie diabético por injerto de Davis. Revista cubana de Angiología y Cirugía Vascular, 18(1).

Díaz R., J. A. (2017). Lipo es cultura. Revista Colombiana de Cirugía Plástica y Reconstructiva, 23(2), 6-7.

Dieppa Ramírez, O. A., \& Alba Matos, E. (2013). Utilidad de la mastoplastia reductiva y su evolución a las técnicas de mínima incisión. Revista electrónica Zoilo E. Marinello Vidaurreta, 38(IV).

Escobar vega, h., Zaldain Ramos, D., Novo, J., Tamayo Carbon, A., \& Exposito Jalturin, A. (2020). Aplicaciones del plasma rico en plaquetas en cirugía estética: revisión de la literatura. Acta Médica, 21(2).

Mejía, C. A. (2020). Cirugía plástica: arte humanista o biotecnología. Revista Colombiana de Cirugía Plástica y Reconstructiva, 26(1).
Patiño Z., W., Cedeño G., J., Sánchez V., A., \& Berruz A., S. (2018). Autoimagen corporal y procedimientos de cirugía plástica estética reconstructiva. Revista Científica Mundo de la Investigación y el Conocimiento, 2(1), 658-573.

Rocca, D. B., Mackfarlane, M., Artero, G., Martínez, M., \& Pefaure, J. (2020). Utilización de colgajo radial para cobertura de exposición protésica en craneoplastias. Revista Argentina de Cirugía Plástica, 26(2), 61-67.

Telich-Tarriba, J., Ramírez-Sosa, L., Palafox, D., Ortega-Hernández, E., \& Rendón-Medina, M. (2020). Aplicacion de la impresion 3D en la cirugía plástica reconstructiva. (U. N. Colombia, Ed.) Revista de la Facultad de Medicina, 68(4).

Vallarta, A., Morales, J., \& A, D. y. (2015). La cirugía plástica y su labor humanitaria en México. Cirugía plástica Ibero-Americana, 457-467.

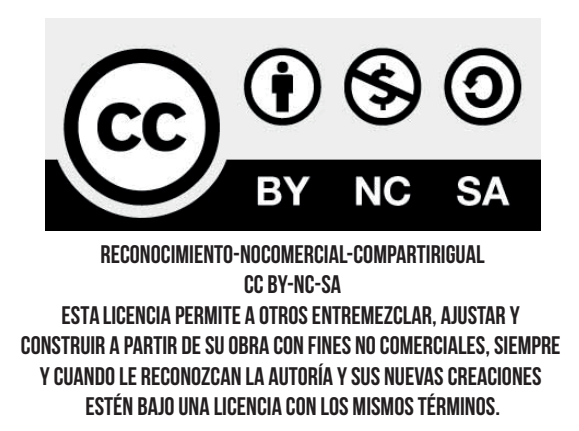

\section{CITAR ESTE ARTICULO:}

Loor Valenzuela, F., Rivas Cáceres, S., Chang Alvarado, P., \& Galindo Veliz, A. (2020). Nuevas tendencias en la cirugía plástica reconstructiva. RECIAMUC, 4(3), 158-166. doi:10.26820/reciamuc/4.(3).julio.2020.158-166 